\title{
Disclosing Jackson Pollock's palette in Alchemy (1947) by non-invasive spectroscopies
}

\author{
F. Rosi , C. Grazia ${ }^{1}$, R. Fontana ${ }^{3}$, F. Gabrieli ${ }^{1}$, L. Pensabene Buemi ${ }^{4}$, E. Pampaloni ${ }^{3}$, A. Romani ${ }^{1}{ }^{12}$, C. Stringari ${ }^{5}$ \\ and C. Miliani ${ }^{1,2^{*}}$
}

\begin{abstract}
Alchemy (1947, Peggy Guggenheim Collection, Venice) is one of the most materic works by J. Pollock, whose palette is extensive, ranging from white to yellow, red, green, violet, blue, black, and silver. Each layer of color was laid on top of a previously dried one and effectively separated from the lower one forming a quite complex stratigraphy with colors intersecting each other. In this study, a non-invasive multi-technique method combining point analysis with Vis-NIR multispectral imaging has been exploited to give insights on the painting technique of the American abstract expressionist. The molecular identification of pigments, colorants and extenders contained in fifteen different paints has been achieved combining key spectral markers from elemental, electronic and vibrational spectroscopies. For those colors exhibiting similar hues but different chemical compositions, a mapping procedure based on false color rendering, obtained by properly mixing three spectral planes from the Vis-NIR multispectral imaging set, has been successfully applied. Relevant for the understanding of the evolution of Pollock's drip technique is the identification of both traditional oil-based paints and oil-modified alkyd media. Point analysis by reflection FTIR scattered throughout the painting enabled mapping the distinct use of traditional and new binding media among painted, squeezed and dripped paints.
\end{abstract}

\section{Background}

In the last decades, many efforts have been devoted to the development of non-invasive and mobile spectroscopic methods based on point analysis and hyper(multi)-spectral imaging $[1,2]$. The results of the most recent in situ application of non-invasive methodologies have shown how the integration of data from different spectroscopic techniques can successfully overcome the intrinsic limitation of each single method and provide valuable information in the study of a variety of heritage objects without sampling [3-5].

The mobile laboratory MOLAB is a collection of integrated mobile instruments based on a variety of noninvasive spectroscopic methods exploiting light-matter interactions (reflection, scattering and emission) in

\footnotetext{
*Correspondence: costanza.miliani@cnr.it

1 CNR-ISTM, Istituto di Science e Tecnologie Molecolari, Perugia, Italy Full list of author information is available at the end of the article
}

different spectral ranges, (from infrared to X-ray), and with different detection methods (point analysis and multispectral imaging) $[1,6]$. In this study, the multitechnique approach of MOLAB has been called to face an unusually challenging task: identifying the palette of the American abstract expressionist J. Pollock, in his iconic painting Alchemy (1947, Peggy Guggenheim Collection, Venice-Additional file 1. Alchemy represents an important development in Pollock's work as it is one of the earliest poured paintings, executed in the revolutionary technique that was his most significant contribution to twentieth-century art [7]. Pollock's movements and directions were disparate and resulted in a stratified pictorial surface with intricate patterns of lines and colors. Alchemy is also one of the most materic works by Pollock, whose palette is extensive, ranging from white to yellow, red, green, violet, blue, black, and silver. The stratigraphy of the painting is quite complex, with colors intersecting each other. The first layer of white, uniformly distributed 
on the canvas, is followed by small drops in blue, yellow and dark red. Only then Pollock begins to pour both black and silver, then bright colors, all smoothly applied in long lines or squeezed directly from the tubes. The lower layers include small quartz fragments (sand and pebbles). Pollock's intention is certainly non-representational and invariably each layer cancels whatever 'figuration' the previous one might have attained. Lines are not meant to describe but simply to exist as traces of movements on the canvas.

The technical study of Alchemy's paints was prompted by its recently concluded conservation project which was aimed at removing both dust and residues that had accumulated on the pictorial surface over time, and penetrated among the cracks and the pigments, and thus made the most vibrant colors look dull and cloudy against the black poured paint. Here we report on the analytical results of the non-invasive technical study of Pollock's palette in Alchemy with a focus on the chemical composition of the wide array of pigments and binders. A more detailed discussion of Alchemy and Pollock's technique, integrating examination and treatment results with the micro-invasive analyses, will be given in a forthcoming publication edited by the Peggy Guggenheim Collection.

\section{Experimental}

Alchemy $\left(114.6 \times 221.3 \mathrm{~cm}^{2}\right)$ was analyzed in situ without moving the painting from its exhibition room and before the restoration process. Vis-NIR multispectral imaging was obtained by scanning the entire surface. A total of more than 300 points of analysis were collected including: about $100 \mathrm{X}$-ray fluorescence spectra, more than 100 FTIR reflection measurements (comprising both near and medium infrared range), more than $80 \mathrm{UV}-$ Vis-NIR reflection and emission spectra, and finally 20 points by Raman spectroscopy performed following the guideline of the previous measurements on a selected number of areas. The selection of points was firstly guided by a visual observation of paint color, texture and morphology. All measurements were digitally documented using the software MOVIDA [8], whose output is an image of the painting reporting all the analyzed points, accessible as an additional file (see Additional file 1).

The following describes the experimental details of the instruments and methods employed.

\section{Multispectral Vis-NIR scanning}

The instrument, specifically designed for the in situ diagnostics of painted surfaces, provides a set of images (16 in the Vis and 16 in the NIR range) that can be analyzed either as a stack of wavelength-resolved images (multispectral Vis-NIR reflectography) or as a series of point reflectance spectra, one for each sampled pixel on the surface (Vis-NIR spectrometry) [9, 10]. The spectral range covered by the instrument is $400-2500 \mathrm{~nm}$, with spectral resolution $20-30 \mathrm{~nm}$ and $50-100 \mathrm{~nm}$ in the Vis and NIR spectral region, respectively. The spatial sampling is 250 micron and the acquisition speed is $3 \mathrm{~h}$ per square meter.

In order to differentiate and map pigments having similar hue but different composition, the monochromatic image planes were properly chosen and jointly processed to produce a false color image. The choice of the most effective bands, governed by the optical properties of the pigment under investigation, was performed on the basis of the reflectance spectra, maximizing the differences between the pigments. Additional file 2 reports all the spectra and bands used to produce false color images.

\section{X-ray fluorescence (XRF)}

XRF measurements were carried out by means of the ELIO spectrometer (XGLab). It consists of a Silicon Drift Detector cooled by a Peltier system, with an active area of $25 \mathrm{~mm}^{2}$ and a resolution of $135 \mathrm{eV}$ to the $\mathrm{K} \alpha$ line of $\mathrm{Mn}$ and a Rhodium source with a maximum power of $50 \mathrm{kV}$ and maximum current of $200 \mathrm{uA}$. The spatial resolution is of about $1 \mathrm{~mm}^{2}$.

\section{UV-Vis-NIR reflection and emission}

The compact portable instrument designed for non-invasive in situ diagnostic described in $[11,12]$ was used for UV-Vis-NIR reflection and emission measurements. The size of the probe is about $2 \mathrm{~mm}^{2}$, corresponding approximately to the achievable spatial resolution. A $266 \mathrm{~nm}$ source (4th harmonic frequency of a pulsed Nd:YAG laser, Alphalas) was used for the zinc oxide fluorescence excitation. Band gap energies were determined from reflectance spectra as described in [11], while emission spectra were processed as described in [12] in order to estimate the composition of cadmium pigments.

\section{Mid and near FTIR reflection spectroscopies}

FTIR spectra were recorded in reflection mode by means of the Alpha spectrometer (Bruker Optics). The measurements were carried out in the $7000-400 \mathrm{~cm}^{-1}$ range at a resolution of $4 \mathrm{~cm}^{-1}$ with 180 scans. The spatial resolution is of about $28 \mathrm{~mm}^{2}$ and the probe head-to-surface distance is more than $1 \mathrm{~cm}$.

Additional measurements were also recorded by the JASCO NIR spectrophotometer equipped with a $2 \mathrm{~m}$ fiber optic probe and working in the range 12500$4000 \mathrm{~cm}^{-1}$ with a spectral resolution of $4 \mathrm{~cm}^{-1}$ and a sampling area of about $12 \mathrm{~mm}^{2}$. 


\section{Raman spectroscopy}

Raman analysis was performed with the portable Xantus-2 by Rigaku. It is equipped with a double laser excitation at 785 and $1064 \mathrm{~nm}$. In both cases, the laser power at the sample can be software modulated thus avoiding any sample damage. The spectral resolution with the $785 \mathrm{~nm}$ excitation is $7-10 \mathrm{~cm}^{-1}$ and it works with a CCD detector (Peltier cooled). An InGaAs detector is employed for the $1064 \mathrm{~nm}$ excitation working with a spectral resolution of $15-18 \mathrm{~cm}^{-1}$. In both configurations the spatial resolution is about $4 \mathrm{~mm}^{2}$.

\section{Results and discussion}

\section{Molecular identification and mapping of colors}

The integrated multi-technique approach permitted the molecular identification of pigments, colorants and extenders contained in the different paints. The key spectral features obtained by point analysis are reported in Figs. 1, 2, 3, 4, 5, 6 (organized by main types of color) along with macro images aimed at documenting the related paint texture and morphology. For those colors exhibiting similar hues but different chemical compositions, a mapping procedure based on false color rendering, obtained by properly mixing three spectral planes from the Vis-NIR multispectral imaging set, has been attempted; the results are shown in Fig. 7 for a specific area of the painting located at the upper-right corner. The corresponding spectra, on whose basis the monochromatic images were chosen, are provided as an additional file (see Additional file 2).

\section{White paints}

Three types of white paints are visible: (i) a cold and opaque white preparation layer, applied with discrete and irregular brushstrokes or a spatula directly on the canvas; (ii) a cold, opaque white with a paste-like consistency, here denoted as white1 (Fig. 1) often seen as final marks direct from the tube or as thin lines; and (iii) a glossy warm, almost cream white (here referred as white2, Fig. 1) having a liquid consistency and appearing as poured paints.

Analyzed by reflection FTIR and XRF, the white ground has been certainly identified as the basic lead carbonate

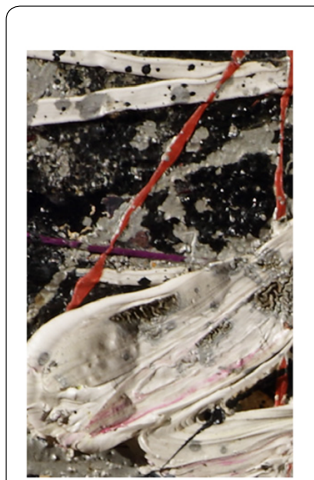

white 1

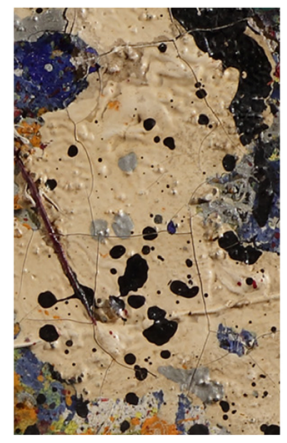

white2
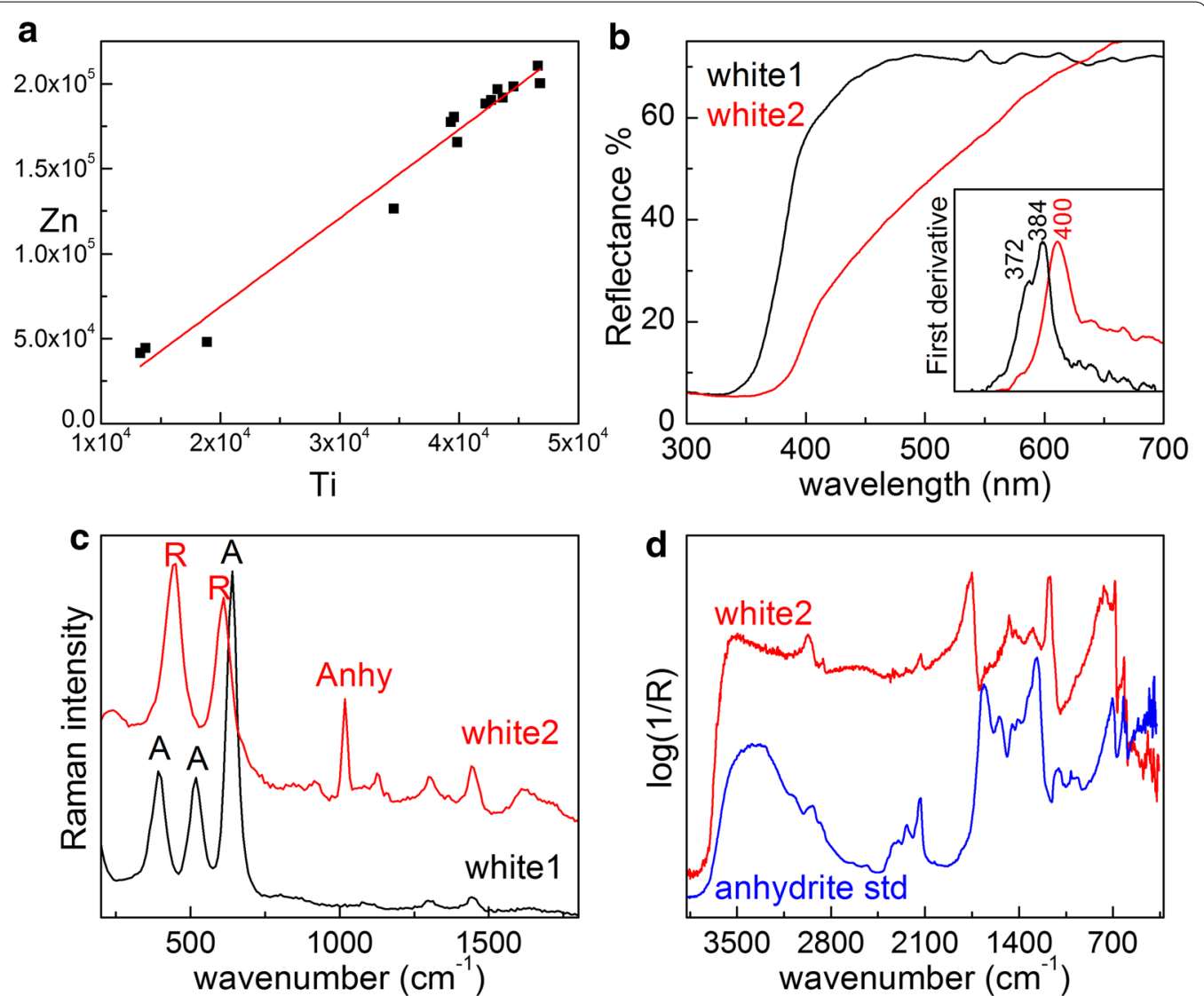

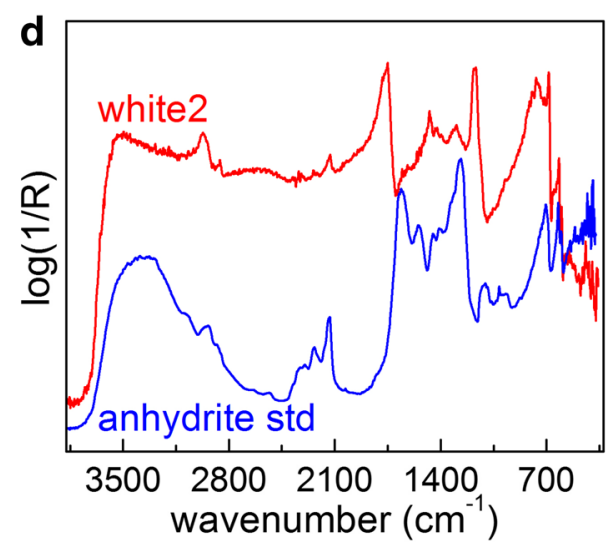

Fig. 1 Details of the visible image and spectral features of paints denoted as whitel and white2. a XRF correlation of the integral of the Zn and Ti $\mathrm{K}_{\mathrm{a}}$ emission lines for white 1 ; b UV-Vis-NIR reflectance spectra and their first derivatives (inset); $\mathbf{c}$ Raman spectra ( $\lambda_{\text {exc }}=1064 \mathrm{~nm}$ ), $R$ rutile, $A$ anatase, Anhy anhydrite; $\mathbf{d}$ FTIR reflection spectrum representative of white2 and compared with the reference spectrum of anhydrite 

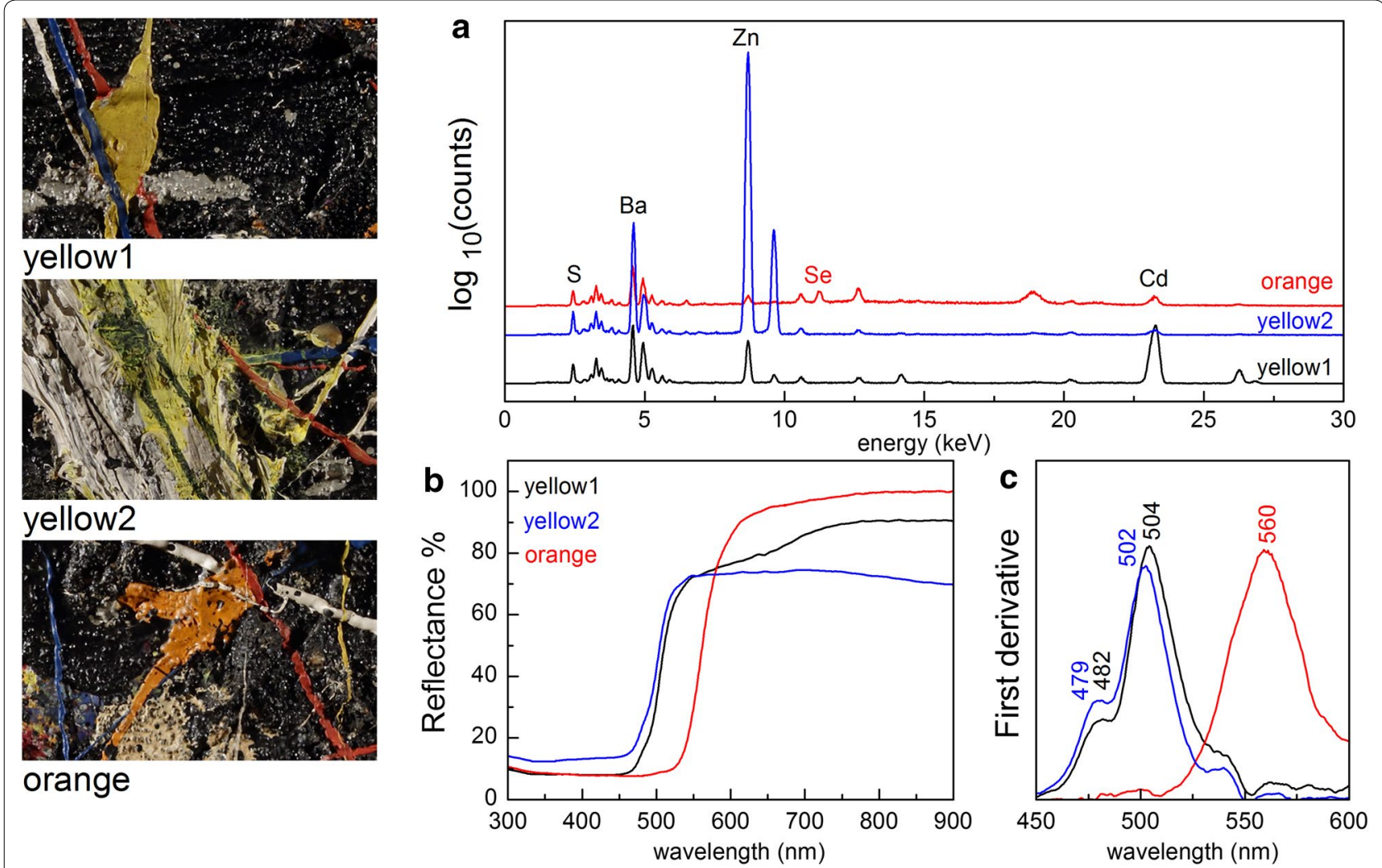

Fig. 2 Details of the visible image and spectral features of paints denoted as yellow1, yellow2 and orange. a XRF spectra; b UV-Vis-NIR reflectance spectra and $\mathbf{c}$ their first derivatives

(lead white).False color image from multispectral VisNIR analysis confirmed that it is mainly visible especially along the margins, where the painting is left at its early stages and fewer layers are applied and both the canvas and the first layer of color are visible (Fig. 7a, b). The white1 elemental composition indicated the presence of a mixture of a titanium and a zinc-based pigment, probably in a ready-made formulation (or a mixture prepared by the artist), as suggested by the fairly constant ratio of the integrated $K_{\alpha}$ emission lines of $\mathrm{Zn}$ and $\mathrm{Ti}$ (Fig. 1a) in all the analyzed areas. Zinc oxide has been ascertained based on the UV-Vis-NIR reflectance profile in Fig. 1b, exhibiting an inflection point at $384 \mathrm{~nm}$ [13] and on the luminescence spectra (data not reported herein) showing the sharp band at around $380 \mathrm{~nm}$ ascribable to $\mathrm{ZnO}$ band gap emission [14] and a broad band at higher wavelengths. The presence of titanium dioxide in the anatase modification, tentatively indicated by the weak inflection point at $372 \mathrm{~nm}$ in the reflectance spectrum (Fig. 1b), has been clearly pointed out by Raman analysis, as shown in Fig. 1c.

Diversely, titanium dioxide in the rutile structure has been detected in the white 2 paint areas by combining UV-Vis-NIR reflection [13] and Raman scattering analysis (Fig. 1b, c). White2, is also characterized by the presence of anhydrite, the anhydrous form of calcium sulfate $\left(\mathrm{CaSO}_{4}\right)$,whose distinctive infrared signals (the $\mathrm{SO}_{4}^{2-}$ combination bands in the range $2300-2000 \mathrm{~cm}^{-1}$, as well as the bending modes in the $700-600 \mathrm{~cm}^{-1}$ range [15]) have been clearly identified in the FTIR spectrum reported in Fig. 1d (red line) where it is compared with a reference spectrum of anhydrite (blue line).

\section{Yellow and orange paints}

Alchemy's yellows are in general associated with final applications and can be visually discriminated in a yellow 1 type, a thick paste paint, and a yellow 2 type, a liquid and transparent paint applied in different degree of thinning over white, silver or black areas (Fig. 2).

$\mathrm{XRF}$ analyses on yellow1 and yellow2 have revealed elemental compositions typical of cadmium-zinc-sulfides (Fig. 2a). Slightly variations in the ratio of $K_{\alpha}$ emission lines of $\mathrm{Cd}$ and $\mathrm{Zn}$ were observed, although they cannot be directly related to a different composition due to possible interferences from white1 areas (which contain $\mathrm{ZnO})$.

Barium sulfate has been identified by reflection FTIR, as the extender of both yellow1 and yellow2. UV-Vis-NIR 


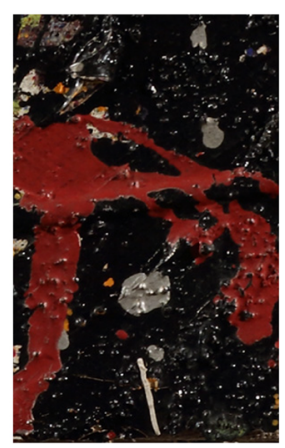

red1

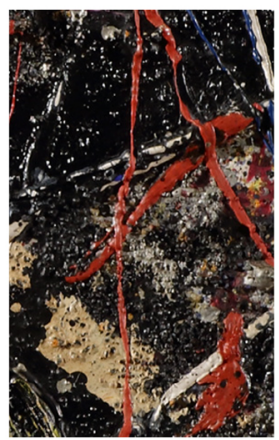

red2
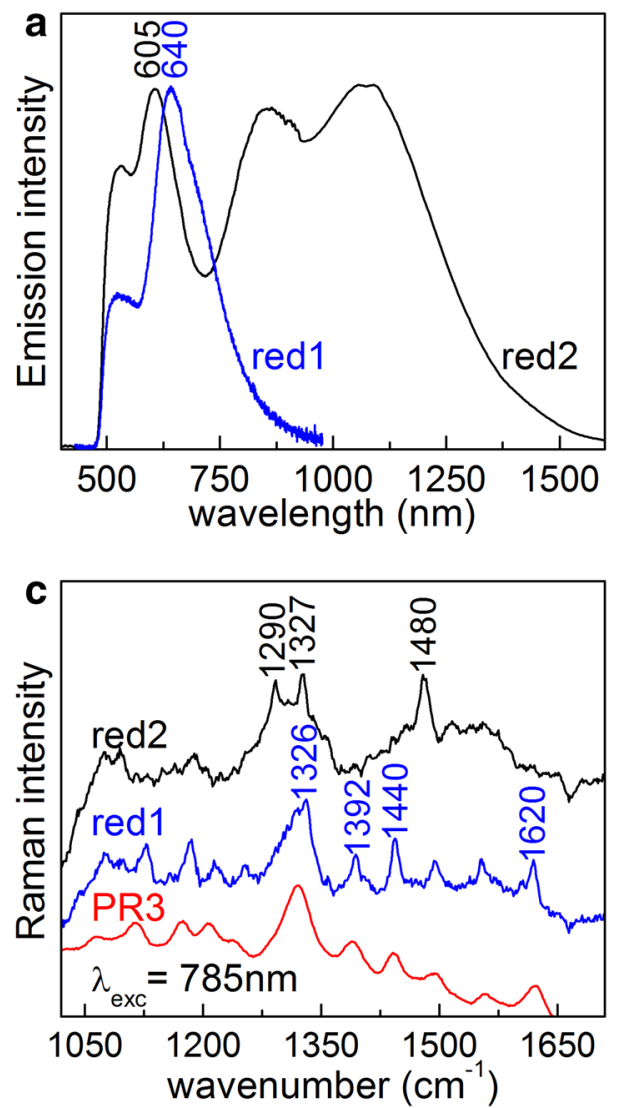
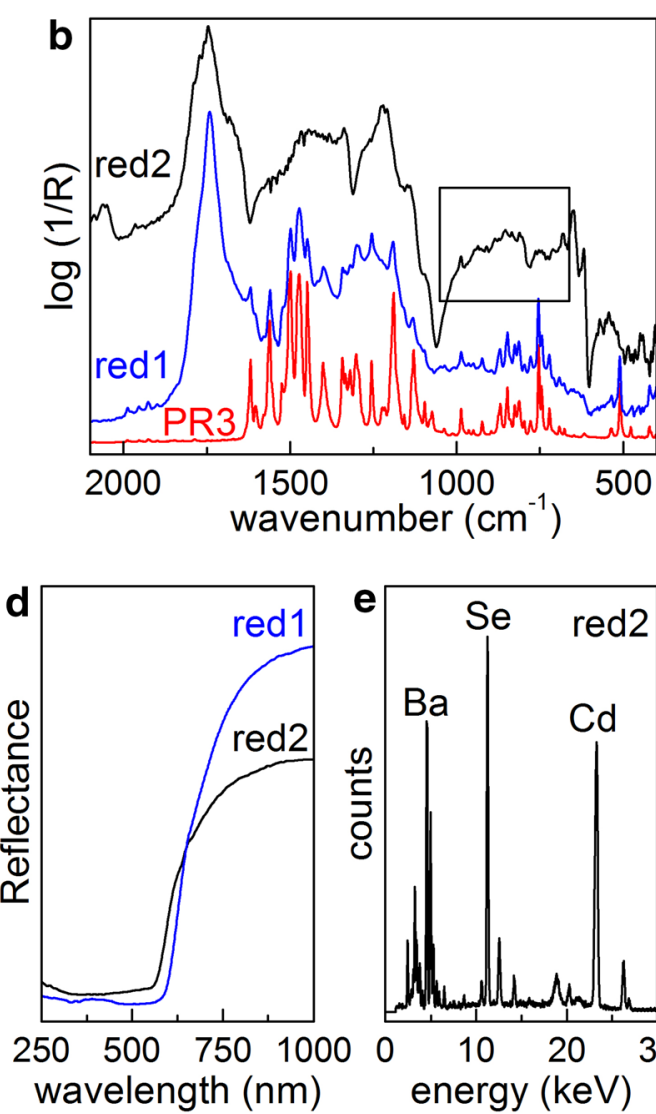

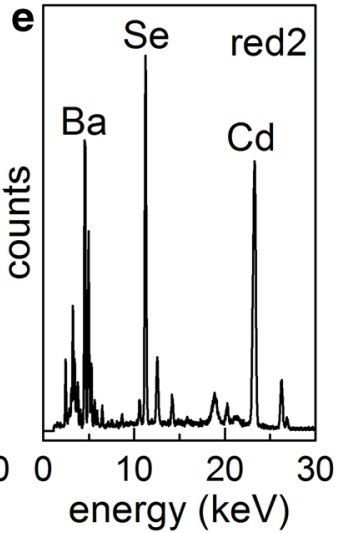

Fig. 3 Details of the visible image and spectral features of paints denoted as red1 and red2. $\mathbf{a}$ UV-Vis-NIR emission spectra $\left(\lambda_{\text {ex }}=445 \mathrm{~nm}\right) ; \mathbf{b}$ FTIR spectra compared with the reference spectrum of PR3 (the rectangle evidences the small signals possibly related to an organic dye on red2); $\mathbf{c}$ Raman spectra $\left(\lambda_{\text {exc }}=785 \mathrm{~nm}\right.$ ) compared with the reference spectrum of PR3 (the Raman spectrum recorded from the PR3 standard was acquired with a portable device having a lower spectral resolution with respect to the system here employed, the laser excitation line is $785 \mathrm{~nm}$ ); $\mathbf{d} \mathrm{UV}$-VisNIR reflectance spectra and $\mathbf{e}$ XRF spectrum representative of red2

reflectance spectra presented in Fig. 2b show the typical sigmoidal shape of semiconductors and do not evidence substantial difference between yellow1 and yellow2. A closer examination of the first derivative spectra collected on both yellow1 and yellow2 areas (Fig. 2c) has highlighted two maxima, possibly indicating a mixture of two different $\mathrm{Cd}_{1-\mathrm{x}} \mathrm{Zn}_{\mathrm{x}} \mathrm{S}$ solid solutions with slightly different zinc contents which however cannot be resolved. The average zinc fraction has been determined to be of about 0.15 , exploiting the linear dependence of the optical band gap with the solid solution stoichiometry as reported in [11]. Very weak luminescence bands (data not shown) were detected in the red-NIR region for both the yellows and ascribed to deep level emissions (DLE) [11]; these signals although too low to be used for confirming the stoichiometry, can be considered to further underline the similarity between the chemical composition of pastose and diluted Cd-based yellows. Thus, non-invasive data (XRF and UV-Vis-NIR reflection and emission) agreed indicating that the pigment and filler of yellow1 and yellow2 are the same, at least within the sensitivity limit of non invasive methods.

As for the orange color (which mainly appears as a thick paint, Fig. 2), the use of a $\mathrm{CdS}_{1-\mathrm{x}} \mathrm{Se}_{\mathrm{x}}$ solid solution with $\mathrm{x}_{\mathrm{Se}}$ of about 0.14 has been inferred on the basis of the optical UV-Vis-NIR reflection and emission properties (Fig. 2b, c) [12].

\section{Red paints}

Two types of red paints having a similar hue can be visually discriminated across the painting on the basis of the surface texture and morphology: a glossy and very liquid paint applied by pouring and flowing onto the bottom edge, here denoted as red 1 and an opaque and thick paint applied as long and thin lines, here denoted as red2 (Fig. 3).

Red1 paint areas are characterized by XRF spectra without any key elements (data not reported herein) and UV-Vis-NIR emission spectra with a rather intense band at about 620-640 nm (Fig. 3a) thus suggesting the use of 

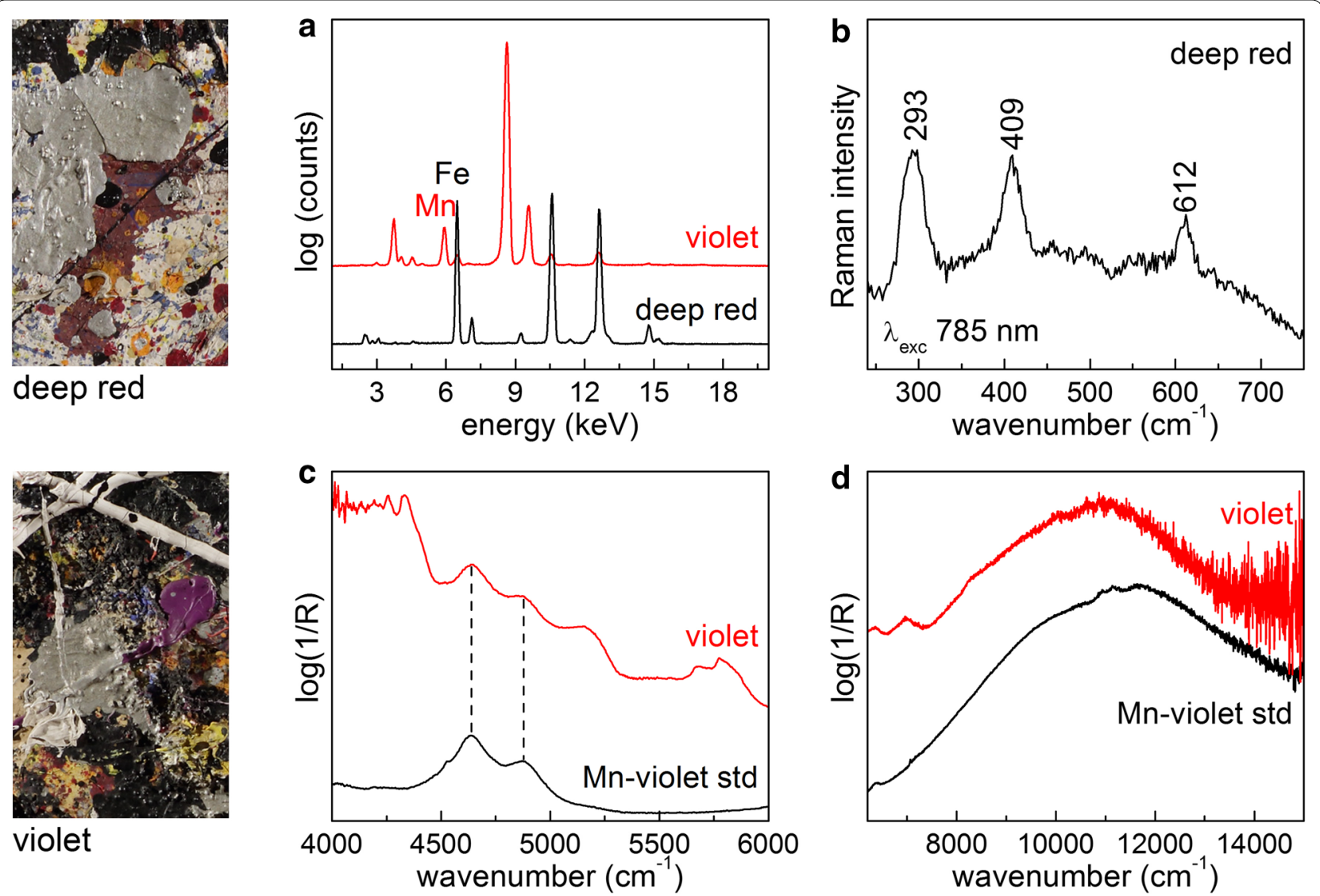

Fig. 4 Details of the visible image and key spectral features of deep red and violet paints. a XRF spectra; $\mathbf{b}$ Raman spectrum of deep red $\left(\lambda_{\text {exc }}=785 \mathrm{~nm}\right) ; \mathbf{c}$ and $\mathbf{d}$ near-FTIR spectra of a violet paint compared with those of standard manganese phosphate

an organic pigment. More conclusively, FTIR spectra collected from the same areas show several distinctive features all closely resembling those of the azo $\beta$-naphthol pigment Toluidine red (also known as PR3), as reported in Fig. 3b. The identification has been confirmed by Raman spectroscopy which gave spectral profiles (Fig. 3c) consistent with that of PR3 [16]. In the painting Mural (1943, University of Iowa Museum of Art, Iowa City),the use of PR3 in mixture with vermillion was also suggested by Gelardi et al. [17] by Py-GC/MS. The reflectance profile (Fig. 3d) shows a strong absorption in the blue-green region and an inflection point at $625 \mathrm{~nm}$; although rather unspecific, those spectral features are compatible with the reference PR3 spectrum reported in [18].

Both XRF and UV-Vis-NIR reflection and emission spectroscopies have inferred that red2 paint areas contain a $\mathrm{CdS}_{1-x} \mathrm{Se}_{\mathrm{x}}$ pigment (Fig. 3a, $\mathrm{d}$ and e). Following the method reported in [12], a deeper examination of the optical band gap has revealed the pigment stoichiometry with a selenium molar fraction of about 0.31 . The identification has been supported by the emission profiles in the red-NIR range (Fig. 3a, black line) showing two DLE bands [12]. From the energy position of the first DLE band, the Se molar fraction has been estimated to be of about 0.33 . All the emission spectra recorded on red 2 areas are characterized by an additional band in the visible range $\left(\lambda_{\max }=605 \mathrm{~nm}\right)$ which has tentatively suggested the presence of an organic pigment different from PR3 (Fig. 3a, blue line), although it could be also due to the emission of the binder partially filtered by the cadmium red [19]. The Raman spectrum recorded on a red2 paint from the edge reported in Fig. 3c (black line), shows some weak bands (over a strong fluorescence background) at about 1290, 1327 and $1480 \mathrm{~cm}^{-1}$ which has allowed to confirm the presence of an organic dye, tentatively identified as anthraquinone-based compound [20]. FTIR spectra recorded on $\mathrm{Cd}$ - and Se-rich areas indicated strong signals of $\mathrm{BaSO}_{4}$ (reststrahlen asym stretching bands at ca. $1070 \mathrm{~cm}^{-1}$ and derivative bending modes in the range $700-600 \mathrm{~cm}^{-1}$ ) and very weak and sharp signals that although not assignable may be related to the organic pigment (Fig. 3b, black line; region highlighted by the rectangle). An anthraquinone organic pigment, Alizarin Crimson lake (PR83), was found in Mural in pink 

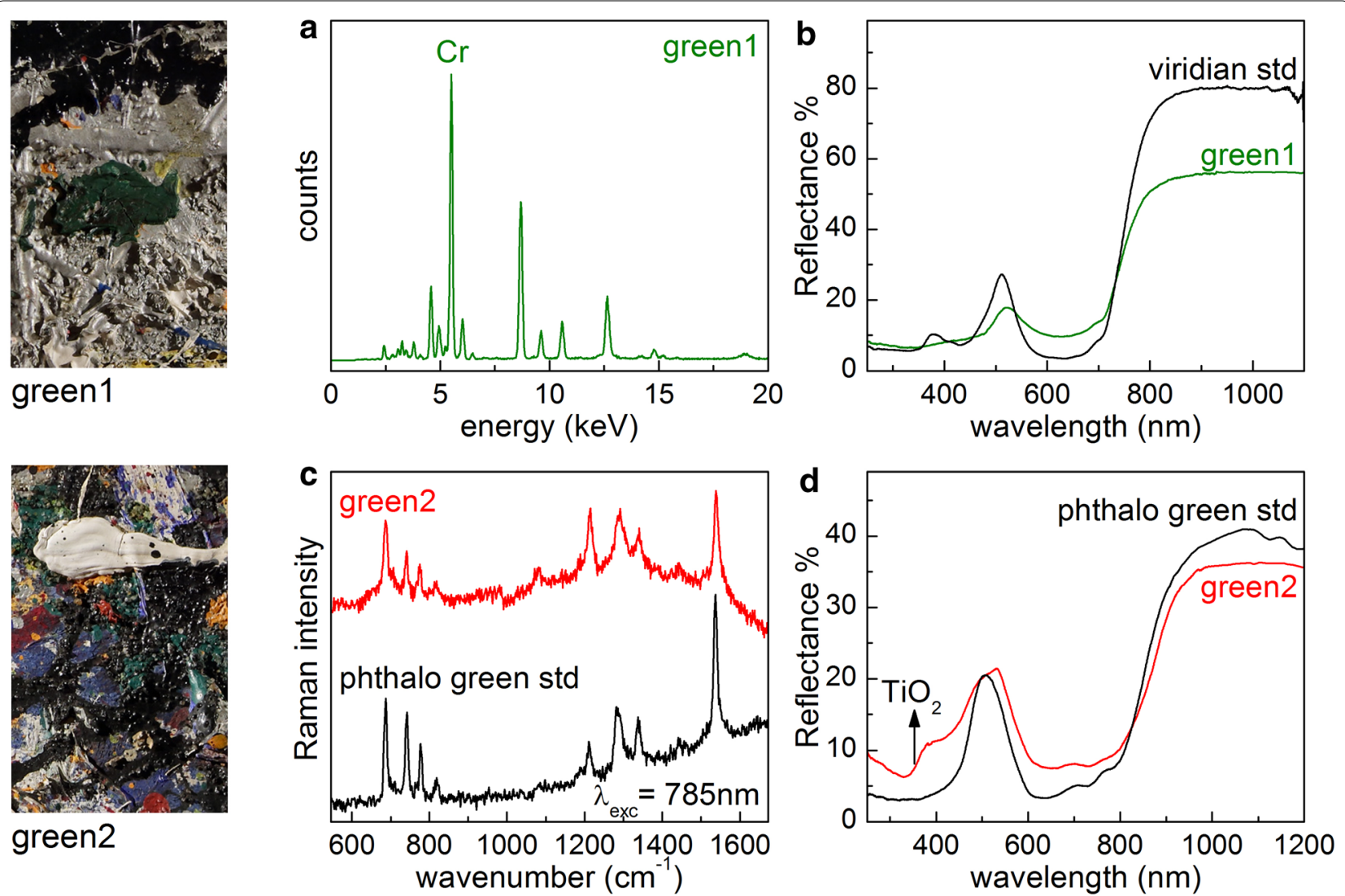

Fig. 5 Details of the visible image and key spectral features of paints denoted as green 1 and green2. a XRF spectrum representative of green 1 ; $\mathbf{b}$ UV-Vis-NIR reflectance spectrum representative of green 1 compared with a reference spectrum of viridian (differences at short-wavelengths could be due to a not identified pigment/extender absorbing in this spectral region; c Raman ( $\lambda_{\text {exc }}=785 \mathrm{~nm}$ ) and $\mathbf{d}$ UV-Vis-NIR reflectance spectra representative of green2, both compared with reference spectra of a phthalocyanine green standard

paint, whilst within the red Cd-based paint a $\beta$-naphtol lake (PR51) was pinpointed [21].

The differences between the reflectance profiles of red 1 and red2 (Fig. 3d) have been exploited to map the distribution of the two paints. In the false color image, shown in Fig. 7c, the discrimination between the two reds is straightforward.

\section{Deep red and violet paints}

Although not so widespread, also dark deep red and bright violet tonalities are visible across the painting, appearing as opaque and flat paints and thin dripping lines, respectively. As shown in Fig. 4a deep red and violet paints are characterized by a different elemental composition, being the first rich in iron and the latter in manganese. In Fig. 4b, an example of the scattering Raman response of deep red areas is shown where, albeit the $A_{1 \mathrm{~g}}$ mode at about $220 \mathrm{~cm}^{-1}$ is not accessible through the portable device, the combined presence of bands at 293, 409 and $612 \mathrm{~cm}^{-1}$ indicates that hematite is responsible for the color in the deep red paints [22,
23]. Near-FTIR revealed the molecular identification of the violet pigment. In this spectral range, the vibrational features at 4640 and $4870 \mathrm{~cm}^{-1}$ (assigned to $v_{1}+v_{4}$, and $v_{2}+v_{3}$ combination bands of ammonium ion) and the electronic $d-d$ transition (at about $11,000 \mathrm{~cm}^{-1}$ ) due to the tetragonal distortion of the $\mathrm{Mn}^{3+}$ octahedral site (Fig. 4c, d) indicate the use of Mn-phosphate manganese violet $\left(\mathrm{NH}_{4} \mathrm{MnP}_{2} \mathrm{O}_{7}\right)$ [24].

False color analysis from the multispectral VIS-NIR data set (Fig. 7d) highlighted that ochre is mainly visible around the edges of the canvas where the painting is left at its early stages, mainly applied directly on the lead white ground. Diversely, manganese violet is painted as final applications of both thin swirls and small patches of full-body paint.

\section{Green paints}

Although at a visual observation the green paints appear to have a similar hue and texture/morphology, thanks to a large number of point measurements collected across the painting surface, two different formulations have been 

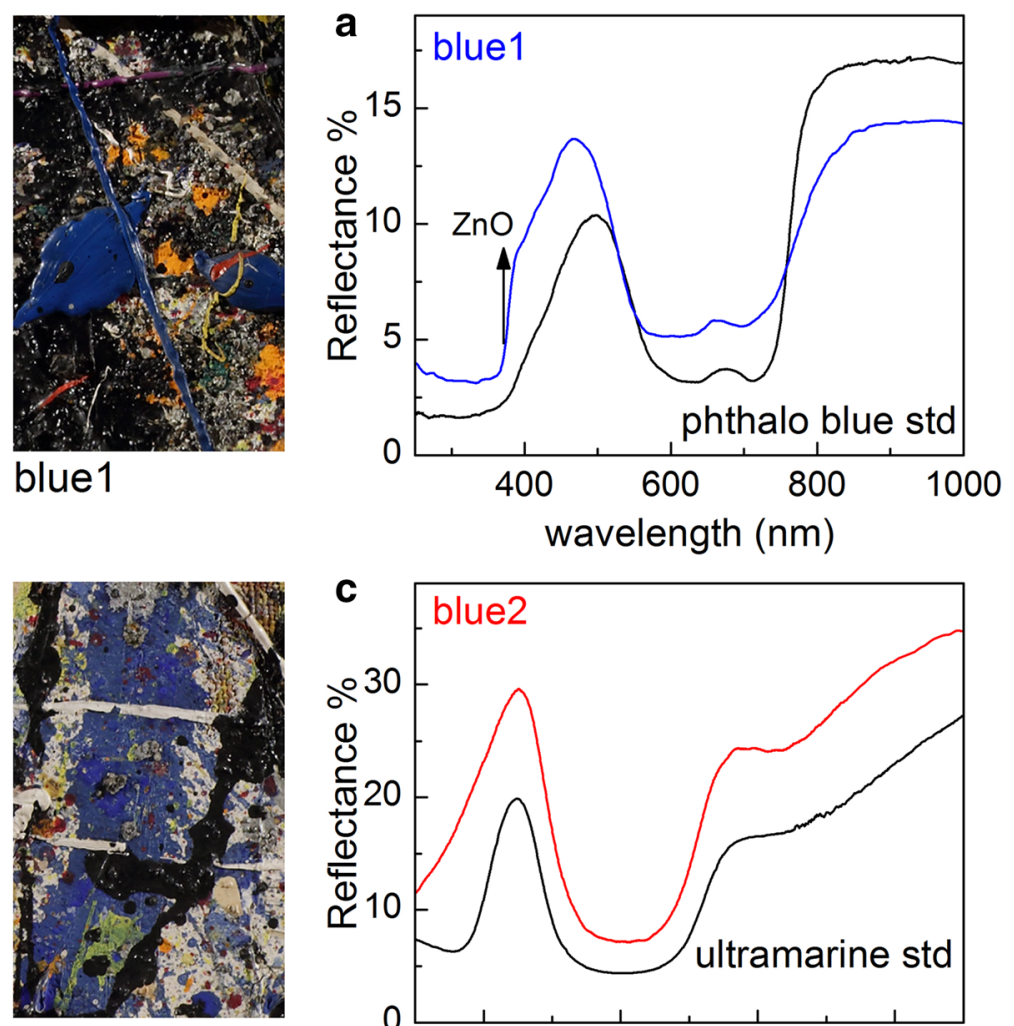

blue2

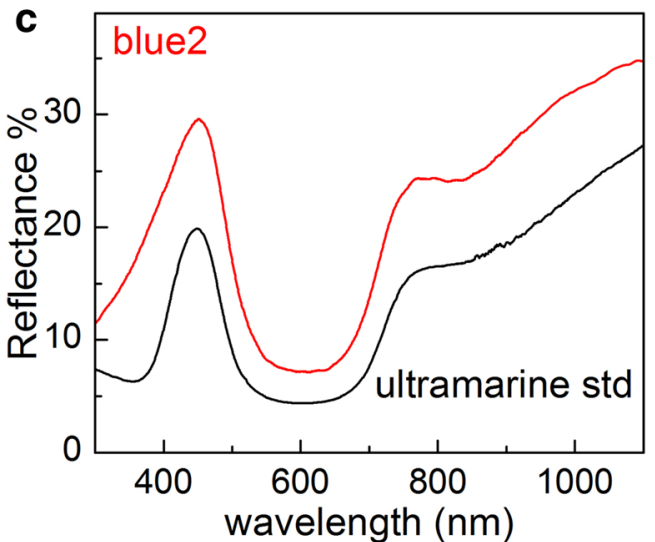

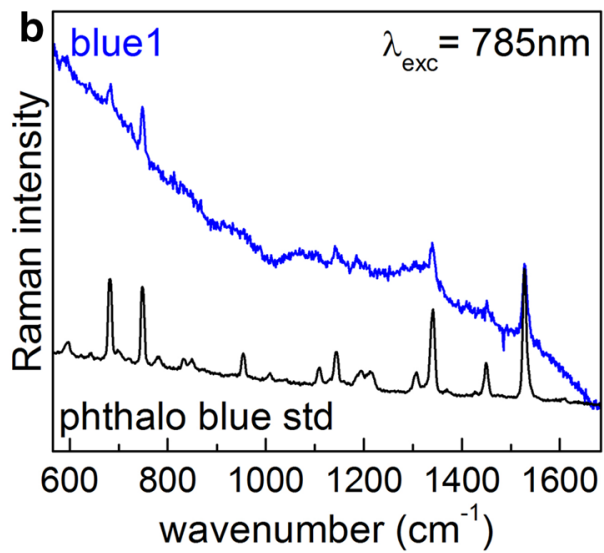

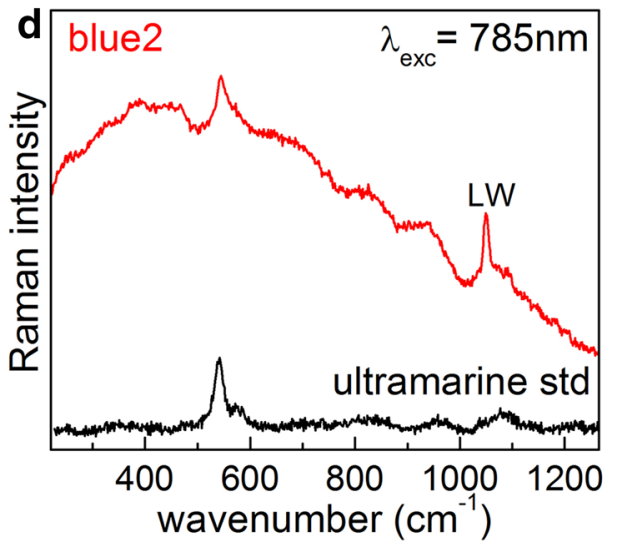

Fig. 6 Details of the visible image and key spectral features of paints denoted as blue 1 and blue2. a UV-Vis-NIR spectrum and $\mathbf{b}$ Raman spectrum $\left(\lambda_{\text {exc }}=785 \mathrm{~nm}\right.$ ) representative of bluel compared with the corresponding reference spectra of a phthalocyanine blue; $\mathbf{c}$ UV-Vis-NIR spectrum and $\mathbf{d}$ Raman spectrum $\left(\lambda_{\text {exc }}=785 \mathrm{~nm}\right.$ ) representative of blue2 compared with the corresponding reference spectra of ultramarine blue

pinpointed. On some of the green paints, XRF measured strong signals of chromium (Fig. 5a), thus indicating both the possible use of chrome green or viridian (respectively $\mathrm{Cr}_{2} \mathrm{O}_{3}$ and its hydrated form $\mathrm{Cr}_{2} \mathrm{O}_{3} \cdot 2 \mathrm{H}_{2} \mathrm{O}$ ) as well as $\mathrm{Cr}-$ based yellow in mixture with a blue. The hydrated form of chromium oxide (here indicated as green1) has been conclusively identified thanks to UV-Vis-NIR reflection spectroscopy [18]. An example of UV-Vis-NIR profile recorded on green 1 areas is reported in Fig. 5b in comparison with the reference compound. Another type of green, labelled as green 2 , has been easily identified as phthalocyanine green, probably in mixture with anatase, by analyzing its vibrational and electronic spectral properties [25, 26]: in Fig. 5c, d, examples of Raman and UV-Vis-NIR spectra collected on green 2 areas are compared with those from phthalocyanine green powder. False color analysis from multispectral VIS-NIR data set allowed for mapping the distribution of the two types of green pigments across the entire painting (Fig. 7e).

\section{Blue paints}

Two types of blue paints of similar hue but rather different surface texture are visible: blue1, generally applied as a thick and full-bodied paint, and blue2, applied as a thin paint layer.

Blue 1 has been clearly identified as phthalocyanine blue from both its characteristic UV-Vis-NIR reflectance profile [25, 27] and Raman pattern [27] (Fig. 6a, b). Interestingly, UV-Vis-NIR reflection (Fig. 6a) and fluorescence (not shown) reveal that the signals of phthalocyanine blue are always associated with the presence of $\mathrm{ZnO}$, besides barium sulfate highlighted by FTIR. These findings along with the visual appearance of the blue1 paints (as a thick lines originating from the direct application from tube) suggest that the mixture is a commercial formulation rather than an intentional addition/ mixture by the artist.

Blue 2 is instead composed of ultramarine blue: also in this case, the experimental identification was achieved 

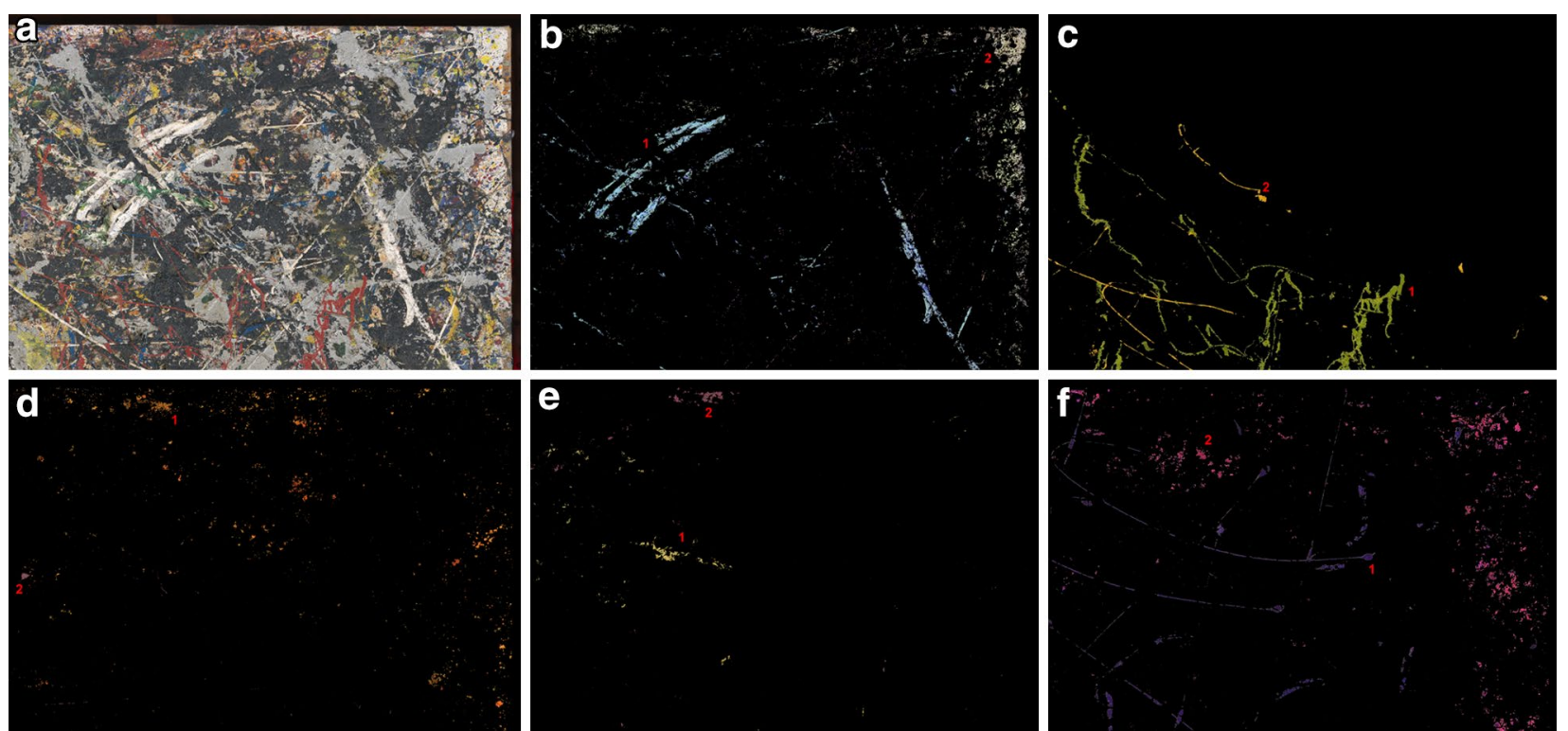

Fig. 7 False color rendering obtained from the Vis-NIR multispectral analysis of the upper right corner of Alchemy. $\mathbf{a}$ visible image; $\mathbf{b}$ distribution of lead white (grey) and whitel (blue) paints $(R=1940 \mathrm{~nm}, \mathrm{G}=1400 \mathrm{~nm}, \mathrm{~B}=590 \mathrm{~nm})$; c distribution of red 1 and red2 paints $(R=765 \mathrm{~nm}, \mathrm{G}=630 \mathrm{~nm}$, $B=455 \mathrm{~nm})$; d distribution of deep red and violet paints $(R=765 \mathrm{~nm}, G=630 \mathrm{~nm}, B=455 \mathrm{~nm}) ; \mathbf{e}$ distribution of green 1 and green2 paints $(R=950 \mathrm{~nm}, \mathrm{G}=765 \mathrm{~nm}, \mathrm{~B}=455 \mathrm{~nm}) ; \mathbf{f}$ distribution of bluel and blue2 paints $(R=765 \mathrm{~nm}, \mathrm{G}=630 \mathrm{~nm}, \mathrm{~B}=455 \mathrm{~nm})$

by UV-Vis-NIR reflection and Raman spectroscopy, as reported in Fig. $6 \mathrm{c}$ and d. Ultramarine blue is often observed along with variable signals of lead white, the blue pigment is in fact often applied over the lead white ground.

False color rendering shown in Fig. 7f, performed by choosing the wavelengths correspondent to differences in the reflectance spectra in the visible region, made it is possible to map the distribution of the two blue colors: ultramarine is more visible as an underlying paint along the edges, while phthalocyanine is a final application of long, thin lines.

\section{Black and silver paints}

As it might be expected, black and silver paints information could be acquired only by XRF, which indicated the use of carbon black and aluminum respectively.

\section{Characterization of binding media}

The characterization of the organic media was accomplished by reflection FTIR which proved to enable the non-invasive identification of the chemical classes for the most commonly used natural and synthetic polymers [28]. It is worth mentioning that FTIR in reflection mode is very sensitive not only to the chemical composition but also to the surface morphological texture. Despite the difficulties of working in reflection mode on surfaces with highly irregular texture as Alchemy's paints, FTIR spectroscopy gave rather consistent and informative results. Only in the case of silver paints the spectra were unreadable being the pseudo absorbance values near to zero within the entire mid- and near-FTIR range.

FTIR data enabled a clear distinction of the glossy black poured paints composed of alkyd-based medium from all the other colors that are bound with drying oil.

Oil paints have been identified by the detection of the specific doublet $\delta+v(\mathrm{CH})$ at about 4340 and $4260 \mathrm{~cm}^{-1}$ (Fig. 8a-e) [28]. A differentiation between oil house paints and artists' oil tubes would be of interest in this context, but is not achievable by FTIR spectroscopy and requires micro-destructive mass spectrometry analysis [29]. As a preliminary step oriented to guide the sampling campaign, non-invasive FTIR spectroscopy enabled us to roughly classify Alchemy's oil-based paints considering signals from additives, alteration products or, more loosely, the entire spectral profile in combination with the visual differences in term of texture, body, and type application of the paints.

As discussed in the previous section, thick-bodied and fluid yellow paints (yellow1 and yellow2, respectively), show a similar inorganic composition based on $\mathrm{Cd}_{1-\mathrm{x}} \mathrm{Zn}_{\mathrm{x}} \mathrm{S}$ solid solution ( $\mathrm{x}$ around 0.15 ) extended with $\mathrm{BaSO}_{4}$. These results and the visual observation of their texture recall the practice reported by Lake et al. for the painting There were even in Eight (1945, MoMA, New York) where cadmium based artists' tube resulted 
"diluted by Pollock to a liquid consistency before dripping them onto the picture surface" [31].

Based on the FTIR spectra recorded on the two yellow areas, a certain identification of the substance(s) possibly added for obtaining the different consistencies is not achievable. Nevertheless, the presence of some other organic components variably visible on spectra recorded in correspondence of yellow paints can be suggested (Fig. 8a, see also Additional file 3 for point location). The spectra recorded on diluted yellow paints (yellow2, spectrum M03) applied over silver areas show FTIR profiles with absorption-like features indicating a transflection mode [30] due to the intense reflection of the infrared light from the metallic paint and its double passing the thin yellow layer. It evidenced the presence of a strong and broad band whose maxima position is at about $1700 \mathrm{~cm}^{-1}$ which in combination with the band at $1470 \mathrm{~cm}^{-1}$ might indicate the presence of a natural resin. A similar spectral profile, even if with a greater contribution of total reflected light since recorded on a thick-bodied yellow paint applied on a black and silver area, is observed for the point M60. The broad band at $1700 \mathrm{~cm}^{-1}$ is observed here as derivative-like band, and the $\mathrm{CH}$ stretching at about $3000 \mathrm{~cm}^{-1}$ are very weak thus suggesting also in this case the possible occurrence of a natural resin. The spectra named M20, M23 and M02 in Fig. 8a are all recorded from yellow1 type paints with a texture resembling the paint directly squeezed from the tube. All of them show, in addition to the lipidic component, two inflection points at about 1700 and $1650 \mathrm{~cm}^{-1}$ which could be related to other organic components. The three spectra (Fig. 8a, M20, M23 and M02 from yellow1 paints) show also very sharp $\mathrm{CH}$ stretching bands at about $3000 \mathrm{~cm}^{-1}$ that, in combination with the sharpness of the bending mode at about $1460 \mathrm{~cm}^{-1}$ and the weak signal at $720 \mathrm{~cm}^{-1}$ (indicated by arrows in the Fig. 8a), reveal the presence of a long chain aliphatic hydrocarbon like beeswax or paraffin. These features are more evident in the spectrum M04 (Fig. 8a, arrows mark the main signal of the (bees)wax-like compound) representative of some yellow1 paints as well as the viridian-based green (green1) as reported in Fig. 8b. Variable amounts of metal oxalates are observed both on the greens and yellows as single band or doublet in the $1400-1200 \mathrm{~cm}^{-1}$ range.

The white1 ( $\mathrm{ZnO} /$ anatase), manganese violet and blue1 (copper phthalocyanine) paints are all characterized by a high content of zinc carboxylates (main bands at 1540 and $1400 \mathrm{~cm}^{-1}$ [32]) and small amount of zinc oxalates (signals at 1360 and $1316 \mathrm{~cm}^{-1}$ [33]) ascribable to saponification and oxidation processes involving zinc oxide and the oil-based medium (Fig. 8c).
White2 (rutile/anhydrite) paints which show a paint flow in the form of smooth and glossy drips, produce spectra where the infrared bands of oil are broadened with respect to that observed for a pure oil binder [28] suggesting the presence of another organic compound with infrared reflection profiles less strong and sharp, perhaps a natural resin (Fig. 8d). Similar profiles were collected for red2 (cadmium sulfide selenide/ $\mathrm{BaSO}_{4}$ ) and phthalo-green-based paints (green2).

Finally, for deep red (hematite), blue2 (ultramarine) and the $\mathrm{Cd}$-based orange, the presence of a lipidic component is evident since the carbonyl stretching at $1740 \mathrm{~cm}^{-1}$ and the $\mathrm{CH}$ combination bands at about $4300 \mathrm{~cm}^{-1}$. The presence of a further broad band in the range around $1700-1600 \mathrm{~cm}^{-1}$ along with the shape of the $\mathrm{CH}$ stretching modes at about $3000 \mathrm{~cm}^{-1}$ (Fig. 8e) may also suggest the addition of another organic component which would require extra investigation by micro-destructive methods to be identified.

Most notably, the black areas, which appear mainly as glossy, smooth/wrinkled and poured paints, are characterized by distinctive and consistent infrared profiles that clearly indicated the use of alkyd-based binding medium (Fig. 8f), a recently introduced synthetic house paint [34]. The direct comparison with a reflectance spectrum acquired on a reference standard of an alkyd enamel shows the similarities of the most characteristic bands: the $\mathrm{C}-\mathrm{O}-\mathrm{C}$ stretching at $1270 \mathrm{~cm}^{-1}$, the $\mathrm{C}-\mathrm{O}$ stretching at $1130 \mathrm{~cm}^{-1}$ and the $\mathrm{C}=\mathrm{C}$ deformation at $1080 \mathrm{~cm}^{-1}$ [28].

It is worth mentioning that similar spectra indicating an alkyd binding medium were also collected from matte black paints, such as the paint used for the artist's signatures (Fig. 8f, see Additional file 4). Only a few spectra recorded from glossy black paints didn't evidence the use of an alkyd resin, revealing only the signals of an oil-based medium, possibly in mixture with a resin (Fig. 8d, black).

In 2004, Lake and co-workers analyzed by GC-MS the binding media of nine of Pollock's poured paintings dating from 1943 to 1950; they reported on the use of artists' oil tubes, oil commercial paints, oleoresin enamels and oil-modified alkyd house paints [31]. Notably, the early use of alkyds was found in Full Fathom Five (MoMA, New York) dated back as Alchemy to 1947 as final splashes of dark blue and orange paints. Extensive use of alkyd house paints was proved in paintings by 1949 $[31,35,36]$ from when it becomes the medium favoured by Pollock for the dripping technique. In 1950 Pollock described his painting method as: "New needs need new techniques. ... Most of the paint I use is a liquid, flowing kind of paint. The brushes I use are used more as sticks rather than rushes .... the brush doesn't touch the surface of the canvas, it's just above" [37]. 

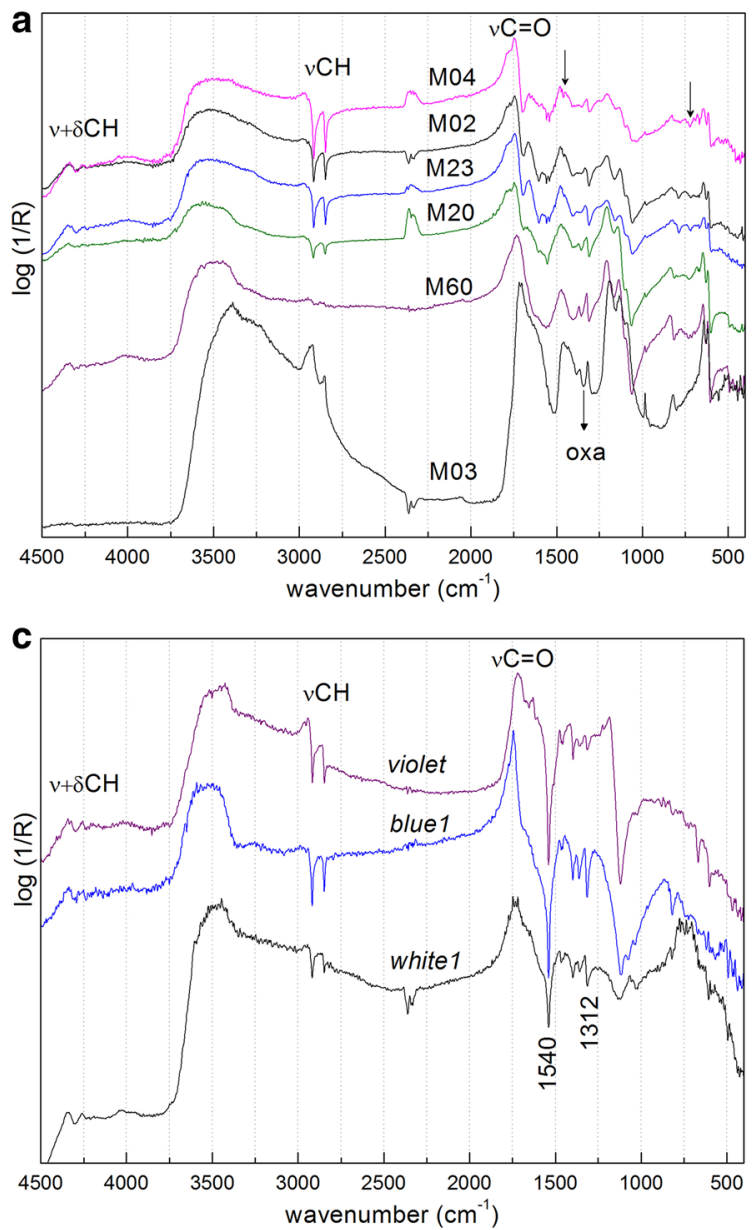

e

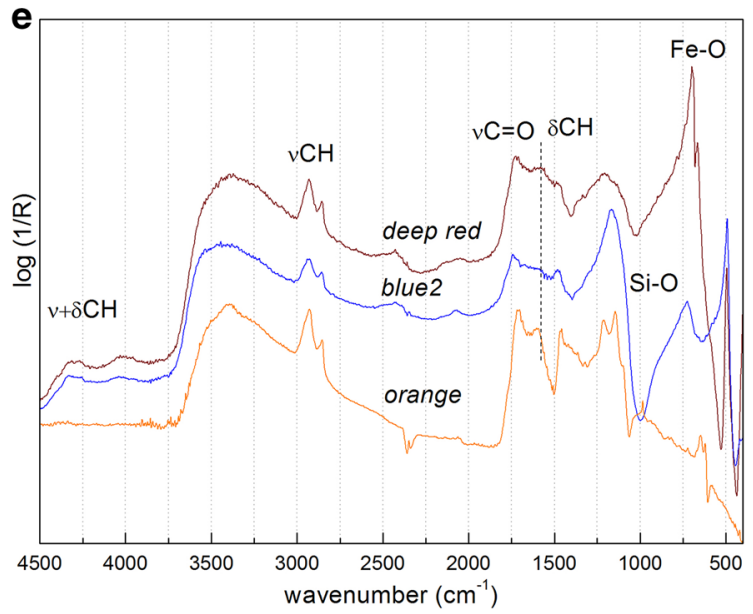

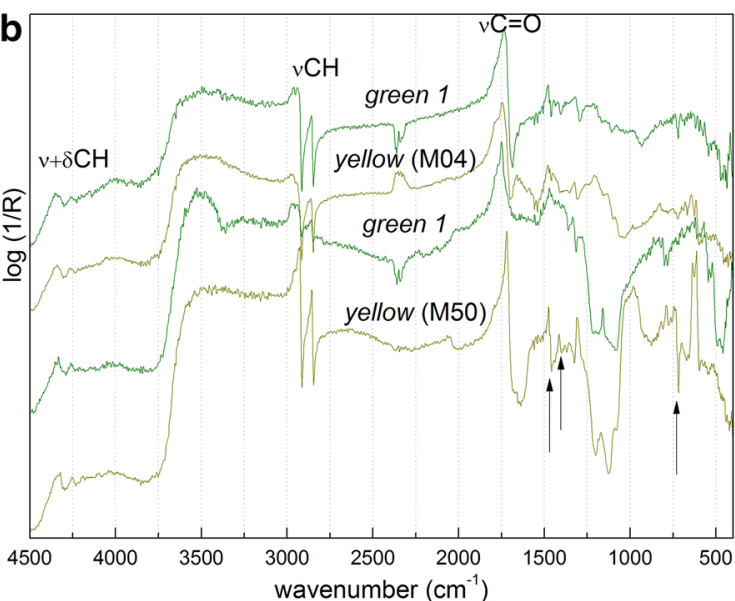
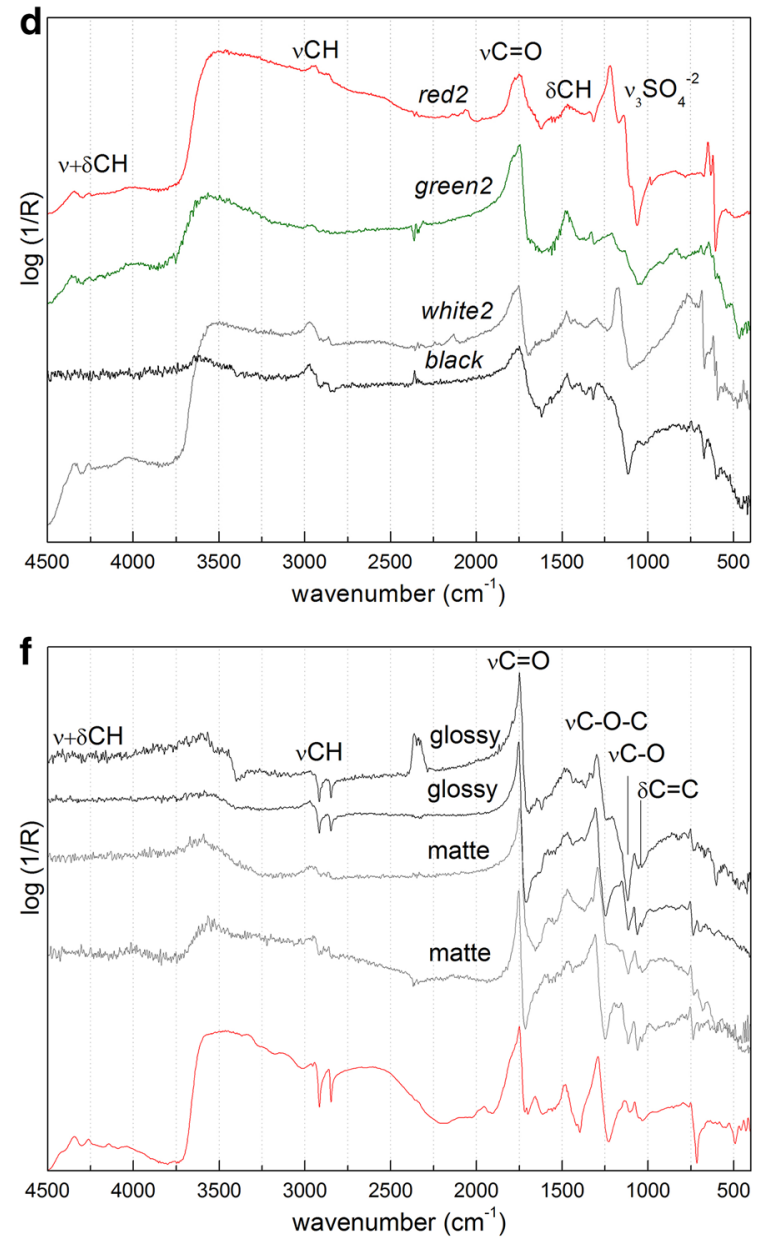

Fig. 8 Reflection mid-FTIR analysis of the Alchemy binding media. a Spectra collected on cadmium yellow paints appearing as thick (yellow 1 ) and diluted (yellow2, M03,) applications (see Additional file 3 for point location); b spectra showing the signals of a long chain aliphatic hydrocarbon characteristic of some yellow paints and the viridian-based green (green 1); c spectra showing the signals of zinc carboxylates and zinc oxalates characteristic of the manganese violet, phthalo blue (blue 1) and $\mathrm{ZnO}$-anatase based white (white1); $\mathbf{d}$ spectra showing the lipidic signals particularly broadened by additional organic compounds characteristic of the red Cd-based (red2), phthalo green (green2), rutile-anhydrite (white2) and some black paints; e spectra showing strong signals from the inorganic constituents of the paint and a further organic component in addition to the lipidone, these profile are characteristic of the hematite-based deep red, ultramarine blue (blue2) and orange CdSSe-based paints; $\mathbf{f}$ spectra from glossy black poured paints (black lines) and black matt paints (grey lines) (see Additional file 4) compared with the spectrum from a reference film of alkyd resin (red line) 


\section{Conclusions}

Alchemy, an intricate and tridimensional network of colors where it is nearly impossible to establish an exact order of materials' application, looked like a true challenge for any technical study. Nevertheless, the non-invasive multi-technique method here employed, profitably combining point analysis with Vis-NIR multispectral imaging, allowed to identify and map fifteen different paints, giving insights into their inorganic and organic components. Lead white and two ready-mixed formulations (rutile/ anhydrite and $\mathrm{ZnO}$ /anatase) were found as white pigments used for different effects and with varied application techniques: lead white applied by brush and spatula directly on the unprimed canvas, rutile/anhydrite as poured glossy paint and $\mathrm{ZnO} /$ anatase as final application directly from tubes. Cadmium sulphide pigments were in yellow, orange and red areas, having chemical composition of $\mathrm{Cd}_{1-\mathrm{x}} \mathrm{Zn}_{\mathrm{x}} \mathrm{S}(\mathrm{x} \approx 0.15), \mathrm{CdS}_{1-\mathrm{x}} \mathrm{Se}_{\mathrm{x}}(\mathrm{x} \approx 0.14)$ and $\mathrm{CdS}_{1-\mathrm{x}} \mathrm{Se}_{\mathrm{x}}(\mathrm{x} \approx 0.32)$, respectively. Cadmium red resulted in mixture with a fluorescent organic pigment, while pure Toluidine red was found on final poured application of fluid red paints. Deep red and bright violet paints were identified as red ochre and manganese phosphate, used in the early stage of the work (directly on the lead white ground layer) and as final application of thin and long lines, respectively. A similar distinction of the order of application emerged for the couple ultramarine blue/ phthalocyanine blue and phthalocyanine green/viridian.

All the bright colored applications resulted to be oilbased paints, with most of the thick-bodied colors being characterized by relevant amount of zinc-carboxylates or long chain aliphatic hydrocarbon possibly indicating the use of artists' tube also considering the paint texture and morphology (opaque and pastose final marks direct from the tube or as thin long lines). The poured paints, such as rutile/anhydride showed infrared profile of an oil-based medium but with broadened bands which suggested the presence of an additional organic component.

Relevant for the understanding of the evolution of Pollock's drip technique is the identification of oil-modified alkyd paint in the black poured areas. To the best of our knowledge, Alchemy (1947), together with Full Fathom Five (1947), is one of the first works where this new medium was adopted by Pollock, indicating 1947 as a year of transition. This finding calls for the analysis of other paintings executed by Pollock during the same period, which could be performed non-invasively by reflection mid-FTIR following the protocol presented in this study.

Even more convincing than in other cases, the noninvasive multi-technique approach appears here, in the case of an extraordinarily complex painting, the first and unavoidable tool to acquire information on the chemistry of the palette toward the understanding of the painting technique.

\section{Additional files}

Additional file 1.jpg image of Alchemy reporting all the areas analyzed by spectroscopic point analyses.

Additional file 2. pdf file reporting for each color mapped, spectrum and wavelengths used for the false color images of Fig. 7.

Additional file 3. jpg image of a portion of the painting reporting point analysis on yellow paints related to Fig. 8a.

Additional file 4. jpg image of the painting signature where black glossy and mat paints are visible.

\section{Authors' contributions}

FR conducted FTIR measurements, analyzed FTIR, Raman and XRF data and drafted the manuscript; CG performed UV-Vis-NIR analysis, interpreted the UV-Vis-NIR data and edited the manuscript; RF interpreted the multispectral data and edited the manuscript; FG conducted Raman and XRF analysis; EP analyzed the multispectral data; LPB conceived the study, interpreted the data and helped to draft the manuscript; AR performed in situ UV-Vis-NIR analysis; CS edited the manuscript; CM supervised the project, the analysis of the data and drafted the manuscript. All authors read and approved the final manuscript.

\section{Author details}

${ }^{1}$ CNR-ISTM, Istituto di Science e Tecnologie Molecolari, Perugia, Italy. ${ }^{2}$ SMAArt, Centro di Eccellenza, Università di Perugia, Perugia, Italy. ${ }^{3}$ CNR-INO, Istituto Nazionale di Ottica, Florence, Italy. ${ }^{4}$ Peggy Guggenheim Collection, Venice, Italy. ${ }^{5}$ Solomon R. Guggenheim Foundation, New York, USA.

\section{Acknowledgements}

This work has been supported by MIUR through the National Access Pilot call (IPERIONCH.it, http://www.iperionch.it) and the PRIN project SICH. Philip Rylands, director of the Peggy Guggenheim Collection (PGC), is acknowledged for giving access to the painting. The MOLAB team is thankful to Siro De Boni and all the staff of the PGC for the logistical support given during in situ analysis. D. Buti and P. Moretti (CNR-ISTM) are acknowledged for XRF in situ analysis, M. Raffaelli (CNR-INO) for in situ Vis-NIR multispectral scanning.

\section{Competing interests}

The authors declare that they have no competing interests.

Received: 15 January 2016 Accepted: 12 May 2016

Published online: 16 June 2016

References

1. Brunetti BG, Miliani C, Rosi F, Doherty B, Monico L, Romani A, Sgamellotti A. Non-invasive investigations of paintings by portable instrumentation: the MOLAB experience. Top Curr Chem. 2016;374:10.

2. Legrand S, Vanmeert F, Van der Snickt G, Alfeld M, de NolfW, Dik J, Janssens K. Examination of historical paintings by state-of-the-art hyperspectral imaging methods: from scanning infra-red spectroscopy to computed X-ray laminography. Herit Sci. 2014;2:13.

3. Van der Snickt G, Miliani C, Janssens K, Brunetti BG, Romani A, Rosi F, Walter P, Castaing J, De Nolf W, Klaassen L. Material analyses of 'Christ with singing and music-making Angels', a late 15th-C panel painting attributed to Hans Memling and assistants: Part I. non-invasive in situ investigations. JAAS. 2011;26:2216-29.

4. Mounier A, Bourdon G, Aupetit C, Belin C, Servant L, Lazare S, Lefrais $Y$, Daniel F. Hyperspectral imaging, spectrofluorimetry, FORS and XRF for the non-invasive study of medieval miniatures materials. Herit Sci. 2014;2:24. 
5. Doherty B, Daveri A, Clementi C, Romani A, Bioletti S, Brunetti BG, Sgamellotti A, Miliani C. The Book of Kells: a non-invasive MOLAB investigation by complementary spectroscopic techniques. Spectrochim Acta Mol Biomol Spectrosc. 2013;115:330-6.

6. Miliani C, Rosi F, Brunetti BG, Sgamellotti A. In situ noninvasive study of artworks: the MOLAB multitechnique approach. Acc Chem Res. 2010;43:728-38.

7. http://www.guggenheim.org/new-york/collections/collection-online/ artwork/3482.

8. Amat A, Miliani C, Brunetti BG. Non-invasive multi-technique investigation of artworks: a new tool for on-the-spot data documentation and analysis. J Cult Herit. 2013;14:23-30.

9. Daffara C, Pampaloni E, Pezzati L, Barucci M, Fontana R. Scanning multispectral IR reflectography SMIRR: an advanced tool for art diagnostics. Acc Chem Res. 2010;43:847-56.

10. Daffara C, Fontana R. Multispectral infrared reflectography to differentiate features in paintings. Microsc Microanal. 2011;17:691-5.

11. Rosi F, Grazia C, Gabrieli F, Romani A, Paolantoni M, Vivani R, Brunetti BG, Colomban P, Miliani C. UV-Vis-NIR and micro Raman spectroscopies for the non destructive identification of $\mathrm{Cd}_{1-x} \mathrm{Zn}_{x} \mathrm{~S}$ solid solutions in cadmium yellow pigments. Microchem J. 2016;124:856-67.

12. Grazia C, Rosi F, Gabrieli F, Romani A, Paolantoni M, Vivani R, Brunetti BG, Colomban P, Miliani C. UV-Vis-NIR and microRaman spectroscopies for investigating the composition of ternary $\mathrm{CdS}_{1-x} \mathrm{Se}_{x}$ solid solutions employed as artists' pigments. Microchem J. 2016;125:279-89.

13. Bacci M, Picollo M, Trumpy G, Tsukada M, Kunzelman D. Non-invasive identification of white pigments on 20th-century oil paintings by using fiber optic reflectance spectroscopy. JAIC. 2007;46: 27-37.

14. Clementi C, Rosi F, Romani A, Vivani R, Brunetti BG, Miliani C. Photoluminescence properties of zinc oxide in paints: a study of the effect of self-absorption and passivation. Appl Spectrosc. 2012;66:1233-41.

15. Miliani C, Rosi F, Daveri A, Brunetti BG. Reflection infrared spectroscopy for the non-invasive in situ study of artists' pigments. Appl Phys A. 2012;106:295-307.

16. Lauridsen CB, Sanyova J, Simonsen KP. Raman analysis of complex pigment mixtures in 20th century metal knight shields of the order of the elephant. Spectrochim Acta Mol Biomol Spectrosc. 2015;150:54-62.

17. Ghelardi E, Degano I, Colombini M, Mazurek J, Schilling M. Learner $\mathrm{T}: P y-G C / M S$ applied to the analysis of synthetic organic pigments: characterization and identification in paint samples. Anal Bioanal Chem. 2015;407:1415-31.

18. Johnston-Feller R. Color science in the examination of museum objects: nondestructive procedures. Getty Conservation Institute; 2001.

19. Thoury M, Delaney JK, Rie ER, Palmer M, Morales K, Krueger J. Nearinfrared luminescence of cadmium pigments: in situ identification and mapping in paintings. Appl Spectrosc. 2011;65:939-51.

20. Schmidt C, Trentelman K. 1064 nm dispersive Raman micro-spectroscopy for the in situ identification of organic red colorants. Preserv Sci. 2009;6:10-21.

21. Szafran Y, Rivers L, Phenix A, Learner T, Landau EG, Martin S. Jackson Pollock's Mural: the transitional moment. Los Angeles: Getty Publications; 2014.
22. De Faria D, Venâncio Silva S, De Oliveira M. Raman microspectroscopy of some iron oxides and oxyhydroxides. J Raman Spectrosc. 1997;28:873-8.

23. Müller M, Villalba JC, Mariani FQ, Dalpasquale M, Lemos MZ, Huila MFG, Anaissi FJ. Synthesis and characterization of iron oxide pigments through the method of the forced hydrolysis of inorganic salts. Dyes Pigments. 2015;120:271-8.

24. Anselmi C, Vagnini M, Cartechini L, Grazia C, Vivani R, Romani A, Rosi F, Sgamellotti A, Miliani C. Molecular and structural characterization of some violet phosphate pigments for their non invasive identification in modern paintings. Spectrochimi Acta Mol Biomol Spectrosc, sent.

25. Poldi G, Caglio S. Phthalocyanine identification in paintings by reflectance spectroscopy. A laboratory and in situ study. Opt Spectrosc 2013;114:929-35

26. Aibéo CL, Goffin S, Schalm O, Van der Snickt G, Laquiere N, Eyskens P, Janssens K. Micro-Raman analysis for the identification of pigments from 19th and 20th century paintings. J Raman Spectrosc. 2008;39:1091-8.

27. Bartolozzi G, Cucci C, Marchiafava V, Masi S, Picollo M, Grifoni E, Legnaioli S, Lorenzetti G, Pagnotta S, Palleschi V. A multidisciplinary approach to the investigation of "La Caverna dell'Antimateria"(1958-1959) by Pinot Gallizio. Herit Sci. 2014:2:29.

28. Rosi F, Daveri A, Moretti P, Brunetti BG, Miliani C. Interpretation of mid and near-infrared reflection properties of synthetic polymer paints for the non-invasive assessment of binding media in twentieth-century pictorial artworks. Microchem J. 2016;124(898):908

29. Kokkori M, Sutherland K, Boon J, Casadio F, Vermeulen M. Synergistic use of Py-THM-GCMS, DTMS, and ESI-MS for the characterization of the organic fraction of modern enamel paints. Herit Sci. 2015;3:30.

30. Bassan P, Lee J, Sachdeva A, Pissardini J, Dorling KM, Fletcher JS, Henderson A, Gardner P. The inherent problem of transflection-mode infrared spectroscopic microscopy and the ramifications for biomedical single point and imaging applications. Analyst. 2013;138:144-57.

31. Lake S, Ordonez E, Schilling M. A technical investigation of paints used by Jackson Pollock in his drip or poured paintings. Stud Conserv. 2004:49:137-41.

32. Otero V, Sanches D, Montagner C, Vilarigues M, Carlyle L, Lopes JA, Melo MJ. Characterisation of metal carboxylates by Raman and infrared spectroscopy in works of art. J Raman Spectrosc. 2014;45:1197-206.

33. Monico L, Rosi F, Miliani C, Daveri A, Brunetti BG. Non-invasive identification of metal-oxalate complexes on polychrome artwork surfaces by reflection mid-infrared spectroscopy. Spectrochim Acta Mol Biomol Spectrosc. 2013;116:270-80

34. Standeven HAL. House Paints, 1900-1960: history and use. Los Angeles: Getty Publications; 2011.

35. Fuesers $\mathrm{O}, \mathrm{Zumbüh} \mathbf{S}$. The influence of organic solvents on the mechanical properties of alkyd and oil paint. In Proceedings of the 9th International Conference on NDT of Art, Jerusalem. 2008.

36. Cappitelli F. THM-GCMS and FTIR for the study of binding media in Yellow Islands by Jackson Pollock and Break Point by Fiona Banner. J Anal Appl Pyrol. 2004;71:405-15.

37. Pollock interview with William Wright for the Sag Harbor radio station, taped 1950 but never broadcast. Reprinted in Hans Namuth, "Pollock Painting", New York 1978, quoted in Crook and Learner, p. 8

\section{Submit your manuscript to a SpringerOpen ${ }^{\odot}$ journal and benefit from:}

- Convenient online submission

- Rigorous peer review

- Immediate publication on acceptance

- Open access: articles freely available online

- High visibility within the field

- Retaining the copyright to your article

Submit your next manuscript at springeropen.com 